\title{
Références bibliographiques du dossier « La lecture en questions »
}

Marie-Geneviève Picart

\section{OpenEdition}

1 Journals

Édition électronique

URL : http://journals.openedition.org/ries/4285

DOI : $10.4000 /$ ries.4285

ISSN : 2261-4265

Éditeur

Centre international d'études pédagogiques

Édition imprimée

Date de publication : 1 juin 1994

Pagination : 98-104

ISSN : $1254-4590$

\section{Référence électronique}

Marie-Geneviève Picart, « Références bibliographiques du dossier « La lecture en questions » », Revue internationale d'éducation de Sèvres [En ligne], 02 | 1994, mis en ligne le 17 avril 2015, consulté le 21 décembre 2020. URL : http://journals.openedition.org/ries/4285 ; DOI : https://doi.org/10.4000/ries. 4285

Ce document a été généré automatiquement le 21 décembre 2020.

(c) Tous droits réservés 


\title{
Références bibliographiques du dossier « La lecture en questions »
}

\author{
Marie-Geneviève Picart
}

1 Cette bibliographie ne prétend pas à l'exhaustivité. Elle signale des références bibliographiques récentes (1990-94). Les documents cités sont disponibles au centre de documentation du CIEP et les quasi-totalités d'entre eux comportent des bibliographies importantes qui complètent celle-ci.

\section{Approches sociologiques et historiques, enquêtes}

2 CHARTIER Roger, dir., Pratiques de la lecture, Paris, éd. Payot et Rivages, Petite Bibliothèque Payot, 1993, $309 \mathrm{p}$.

F. Bresson, P. Bourdieu, R. Chartier, R. Darnton, D. Fabre, J.-M. Goulemot, J. Hébrard, L. Marin, D. Roche, dans le cadre d'une rencontre consacrée à la lecture et à ses pratiques, croisent leurs approches et analysent, à travers des disciplines différentes (l'histoire, la pédagogie, la psychologie...), la pratique culturelle de la lecture. (Édition de poche qui reprend l'édition parue en 1985 aux éd. Rivages.)

DUMONTIER Françoise, SINGLY François de, THELOT Claude, «La lecture moins attractive qu'il y a vingt ans ", Économie et statistique, $n^{\circ} 223$, juin 1990, p. 63-80.

Les Français lisent moins en général, malgré l'extension des loisirs, du degré de scolarisation et de l'accroissement de l'offre de lecture. Le meme constat est appliqué aux étudiants.

ELLEY Warwick B., How in the world do students read?, IEA study of reading literacy, Hambourg, International Association for the Evaluation of Educational Achievement, 1992, 120 p., tableaux.

Cette enquête internationale sur le niveau en lecture s'est déroulée en 1991 dans une trentaine de pays des deux hémisphères, dont tous les pays de l'OCDE, à l'exception du Luxembourg et de l'Angleterre. Deux populations d'élèves ont été interrogées : à 9 ans et à 14 ans. Les textes proposés étaient classés en trois catégories : les documents, les exposés et les narrations. 
FRAISSE Emmanuel, dir., Les étudiants et la lecture, Paris, Presses universitaires de France (Politique d'aujourd'hui), 1993, $263 \mathrm{p}$.

Le ministère de l'éducation a créé en 1991 une mission lecture afin de permettre une réflexion permanente sur la lecture, en liaison avec les professionnels du livre. Les nombreuses contributions réunies ici visent à définir l'identité étudiante dans notre système d'enseignement supérieur, le rôle de la lecture dans les apprentissages universitaires et permettent, à travers l'analyse de l'offre de lecture des bibliothèques universitaires, de programmer des actions et des expérimentations en faveur de la lecture.

6 FRAENKEL Béatrice, dir., Illettrismes : variations historiques et anthropologiques, Paris, BPI Centre Georges Pompidou (Études et recherche), 1993, 305 p.

Cet ouvrage rassemble les contributions au colloque organisé par le Centre d'études de l'écriture (université Paris VII) en mai 1991. La définition de l'illettrisme pose problème. Ce recueil tente de situer le phénomène de l'illettrisme en France tout d'abord, puis en Extrême-Orient, dans des pays caractérisés par l'emploi de l'écriture idéogrammatique. La III partie situe l'illettrisme du point de vue des pratiques d'écriture " ordinaires ", tandis que la dernière se penche sur les usages et les pratiques de l'écrit en situation de travail.

MINISTÈRE DE LA CULTURE ET DE LA COMMUNICATION, Nouvelle enquête sur les pratiques culturelles des Français en 1989, Paris, Documentation française, 1990, 285 p.

Présente les résultats d'une enquête par sondage sur les pratiques culturelles des Français de quinze ans et plus. Données statistiques permettant de mesurer la réalité des changements et laissant apparaître un relatif recul de la lecture chez les jeunes alors que la scolarisation secondaire continue de se développer.

8 POULAIN, Martine, dir., Histoire des bibliothèques françaises: les bibliothèques au XX $\mathrm{X}^{e}$ siècle (1914-1990), Paris, Cercle de la Librairie - Promodis, 1992, 793 p.

Quatrième et dernier tome de l'Histoire des bibliothèques françaises. Synthèse sur les politiques du livre, l'évolution des bibliothèques et l'histoire de la lecture.

POULAIN Martine, dir., Lire en France aujourd'hui, Paris, Cercle de la Librairie, 1993, $255 \mathrm{p}$.

Les contributions réunies dans ce recueil cherchent à dévoiler la pluralité, la complexité et l'inventivité des approches récentes de la lecture ordinaire qui, dès l'avantpropos, est distinguée de la lecture de l'expert. Ses auteurs, historiens, sociologues, littéraires, didacticiens, psychologues nous permettent de mesurer la diversité des comportements de lecture de la France d'aujourd'hui.

PRIVAT Jean-Marie, éd., REUTER Yves, éd., Lectures et médiations culturelles, actes du colloque de Villeurbanne, Lyon, Presses universitaires, 1991, 196 p.

Analyse des distorsions et des contradictions existant fréquemment entre l'offre culturelle instituée et les demandes ou besoins multiformes des lecteurs.

11 De nombreux dossiers Éducation \& formations, publiés par la direction de l'évaluation et de la prospective du ministère de l'éducation nationale exposent les résultats d'enquêtes menées auprès de jeunes en milieu scolaire.

$\mathrm{N}^{\circ} 24$ : de SINGLY François, Les jeunes et la lecture, 1993.

$\mathrm{N}^{\circ} 25$ : de SINGLY François, Matériaux sur la lecture des jeunes, 1993.

$\mathrm{N}^{\circ} 29$ : BRESSOUX Pascal, DESCLAUX Agnès, Mesures incitatives à la lecture: bilan d'une expérimentation à l'école élémentaire, 1993. 
$\mathrm{N}^{\circ} 30$ : BRESSOUX Pascal, Les performances des écoles et des classes : le cas des acquisitions en lecture, 1993.

$\mathrm{N}^{\circ} 33$ : Évaluation CE2-6e : résultats nationaux, septembre 1993.

\section{Approches psychologiques et psycholinguistiques}

CHAUVEAU Gérard, dir., RÉMOND Martine, dir., ROGOVAS-CHAUVEAU Éliane, dir., L'enfant apprenti lecteur : l'entrée dans le système écrit, Paris, L'Harmattan-INRP (collection Cresas ; $\left.\mathrm{n}^{\circ} 10\right), 1993,220 \mathrm{p}$.

Ce livre prend ses distances par rapport aux approches psycholinguistiques classiques de l'enfant apprenti lecteur, pour recentrer cette problématique en englobant le décodage et la compréhension du texte écrit. L'enfant et sa maîtrise de la lectureécriture sont replacés au centre des observations et des analyses. Cet ouvrage, qui présente les recherches de plusieurs équipes, aborde surtout les aspects métalinguistiques et métacognitifs de l'acquisition, dans une perspective cognitive.

FAYOL Michel, GOMBERT Jean-Émile, LECOCQ Pierre, SPRENGER-CHAROLLES Liliane, ZAGAR Daniel, Psychologie cognitive de la lecture, Paris, Presses universitaires de France (Psychologie d'aujourd'hui), 1992, $37 \mathrm{p}$.

Les contributions proposent plusieurs modèles de l'activité de lecture et traitent des mécanismes d'identification, des processus automatisés, des processus contrôlés et des capacités métalinguistiques.

GAONAC'H Daniel, "Les composantes cognitives de la lecture", Le français dans le monde, $\mathrm{n}^{\circ}$ 255, mars 1993, p. 87-92.

Cet article retrace les différents niveaux de traitement en lecture, en langue maternelle et en langue étrangère. La stratégie est identique dans les deux cas et s'effectue en plusieurs étapes : l'appui sur les régularités orthographiques, la maîtrise du lexique, la représentation phonologique et la mémorisation transitive et solide.

JAFFRE Jean-Pierre, dir., SPRENGER-CHAROLLES Liliane, dir., FAYOL Michel, dir., Lecture-Écriture : acquisition, les actes de La Villette, Paris, Nathan (Pédagogie), 1993, $319 \mathrm{p}$.

Le colloque «Acquisition de la lecture/écriture et psychologie cognitive " s'est tenu à La Villette les 30 et 31 janvier 1993. Genèse de l'écriture, aspects linguistiques de l'acquisition de la lecture-écriture, capacités métalinguistiques de l'enfant et compréhension de l'écrit, autant de thèmes majeurs qui permettent ici une confrontation des recherches actuelles.

16 LECOQ Pierre, éd., La lecture: processus, apprentissage, troubles, Lille, Presses universitaires, 1992, $270 \mathrm{p}$.

Ce livre réunit l'ensemble des communications présentées lors du colloque européen sur «La Lecture », organisé à l'université Charles-de-Gaulle de Lille en mars 1990, à l'initiative de la Société française de psychologie. Sont abordés les activités sensorielles et motrices, les processus de traitement et les difficultés spécifiques à la lecture, qu'elles soient liées à des syndromes, à la surdité, ou à la dyslexie. 


\section{Théorie(s) de l'interprétation et de la réception}

PANIER Louis, dir., Le temps de la lecture : exégèse biblique et sémiotique, recueil d'hommages pour Jean Delorme, Paris, éd. du Cerf (Lectio divina), 1993, 391 p.

À l'initiative du CADIR (Centre d'analyse des discours religieux) et de la faculté de théologie de Lyon, quelques amis de Jean Delorme ont rendu hommage dans ce recueil à son travail de recherche biblique où se sont confrontées exégèse biblique et sémiotique. C'est une théorie sémiotique de la lecture qui nous est proposée ici, à travers l'analyse des textes bibliques, par des réflexions théoriques sur les questions d'interprétation et de réception notamment, ainsi que par des exercices pratiques.

JOUVE Vincent, La lecture, Paris, Hachette Supérieur (coll. Contours littéraires), 1993, $112 \mathrm{p}$.

Critique des textes littéraires et théorie de la lecture sont ici rapprochés pour aborder la spécificité de l'œuvre. Peut-on rendre compte de l'acte de lecture, quel est le statut du récepteur, comment se déroule l'interaction texte/lecteur, quel effet la lecture produit-elle sur un individu, sur une société, autant de questions abordées ici de façon claire et précise à travers différentes théories: les théories de la réception (Jauss et Iser), l'approche sémiotique (Eco, Hamon et Otten), et celle de M. Picard centrée sur le lecteur réel. Des extraits d'ouvrages cités illustrent cette introduction à la lecture.

\section{Études de parcours individualisés en milieu scolaire ou non}

HASSENFORDER Jean, dir., Lecteurs et lectures en éducation, Paris, INRP-L'Harmattan (Éducation \& formation), 1993, $357 \mathrm{p}$.

Publiés dans une revue de l'INRP intitulée Perspectives documentaires en éducation, les «Itinéraires de lecture » présentés ici sont des témoignages de professeurs et de chercheurs en sciences de l'éducation (mais non spécialistes de la lecture), acceptant de livrer les lectures qui les ont marqués ou qui leur ont permis d'étayer leur réflexion professionnelle, décrivant ainsi une lecture mosaïque.

KLELTZ Françoise, Étude sur les pratiques universitaires de lecture en sciences humaines et sociales, Paris, ministère de la recherche, syndicat national de l'édition, 1991, 2 vol. , 146 et $360 \mathrm{p}$.

PENNAC Daniel, Comme un roman, Paris, Gallimard, 1992, 173 p.

Plaidoyer pour une pédagogie de la lecture, face à l'illettrisme et à l'échec scolaire. Déclaration des droits imprescriptibles du lecteur: lecture à voix haute, lectures superficielles, sauts de page, romans de gare... Enseignants, parents sont interpellés pour réhabiliter la passion de lire.

PRIVAT Jean-Marie, coord., Pratiques de lecteurs, Pratiques, n 80, décembre 1993.

Les contributions rendent compte ici des pratiques scolaires et sociales des jeunes lecteurs et mettent l'accent surtout sur le travail de médiation culturelle de l'école en proposant des perspectives didactiques : travail en équipes d'enseignants, création de réseaux d'échanges, lectures partagées au sein de la classe et rôle des BCD (Bibliothèques Centres documentaires) dans la construction des compétences en lecture. 


\section{Propositions de stratégies et de pratiques pédagogiques}

ASSOCIATION POUR FAVORISER UNE ÉCOLE EFFICACE, FÉDÉRATION DES FAMILLES DE

FRANCE, La lecture pour tous, Paris, Armand Colin, 1991, $110 \mathrm{p}$.

C'est dans le cadre du colloque "Apprendre à lire» du 15 mai 1993, que la FEE (Fondation de l'école efficace, placée actuellement sous l'égide de la Fondation de France) a lancé son appel pour un savoir lire garanti à tout enfant. Cinq chercheurs examinent ici les contextes d'apprentissage, scolaires, familiaux, socioculturels, décrivent les modes d'appropriation de l'écrit et les compétences effectives en communication, et définissent des perspectives didactiques pour une pédagogie efficace de l'écrit.

BAYER, f.-Y., MATEO, P., SNYDERS, G. et al, «Lecture et écriture », Revue française de pédagogie, 1992, n 99.

Le problème de la lecture et de l'écriture est abordé en six articles qui traitent de la lisibilité d'un texte, de l'apprentissage de la lecture en cycles d'apprentissage, de l'impact pédagogique des BCD, de la pédagogie différenciée en SES (section d'éducation spécialisée).

CORNAIRE Claudette, Le point sur la lecture en didactique des langues, Montréal, Centre éducatif et culturel (Le point sur...), 1991, $127 \mathrm{p}$.

Ce petit ouvrage réalise une synthèse sur l'acte de lire en donnant des éclairages théoriques et fait le point sur les recherches menées dans le domaine de la lecture, en langue maternelle puis en langue seconde. Une importante bibliographie clôt l'ouvrage.

DECREAU Laurence, Ces héros qui font lire, Paris, Hachette Éducation (Pédagogies pour demain), 1994, $143 \mathrm{p}$.

Retrouver le plaisir de la lecture, tel est le slogan de cet ouvrage qui prône l'introduction des séries romanesques au sein de l'école : Aladin, Le Club des Cinq, Alice, Indiana Jones... autant de héros qui accompagnent l'apprenti-lecteur et, tout en constituant une transition vers la littérature, offrent à l'enseignant des pistes pédagogiques intéressantes.

FEITELSON, D., dir., BUS, A.G., dir., «Beginning reading instruction», International Journal of Educational Research, vol. 19, w 7, 1993, p. 601-665.

Ce numéro met en garde contre les conséquences négatives de l'entraînement au décodage et propose de traiter la lecture comme un élément de l'activité écrite (literacy), dès le plus jeune âge.

28 GREGORY Eve, "What counts as reading in the early years'classroom? », British Journal of Educational Psychology, vol. 63, part II, juin 1993, p. 214-230.

Étude de la pédagogie britannique dans l'enseignement primaire, face aux difficultés de lecture des enfants. L'enseignement initial de la lecture est le plus souvent possible individualisé (compte rendu de l'article dans Perspectives documentaires en éducation, $\left.\mathrm{n}^{\circ} 30,1993\right)$.

INSTITUT NATIONAL DE RECHERCHE PÉDAGOGIQUE (INRP), Lecture/écriture: des approches de recherche, Paris, INRP, 1993, $292 \mathrm{p}$.

Cet ouvrage reprend des publications (ouvrages ou articles de revues), sous forme d'extraits significatifs, en privilégiant le caractère récent des contributions. L'éventail 
des textes présentés ici reflète l'importance actuelle des travaux menés à l'INRP sur l'apprentissage de la lecture et de l'écriture de la maternelle à l'université.

PARMEGIANI Claude-Anne, dir., Lectures, livres et bibliothèques pour enfants, Paris, Cercle de la Librairie, 1993, 207 p.

L'édition jeunesse et les bibliothèques pour enfants ont connu un formidable essor. Le contenu des livres pour enfants a évolué, les pratiques d'animation autour de la lecture se sont multipliées; des spécialistes de la littérature de jeunesse rendent compte ici de ces transformations, et s'interrogent sur les rôles respectifs de l'enseignement et des bibliothèques. Une bibliographie sélective des revues de langue française sur le livre et la littérature pour enfants et adolescents complète cet ouvrage.

\section{Évaluation des compétences et des difficultés en lecture}

31 HINCAPIE-JAULT Isabelle, « Lecture en SES », Médialog, nº 19, décembre 1993.

Panorama des logiciels de lecture utilisés pour le soutien scolaire et testés dans le cadre d'une expérience de lecture ayant pour objectif la progression de chaque enfant par des exercices appropriés.

ZAGAR, D., JOURDAIN, C, LETE, B., Logiciel d'évaluation-diagnostic des capacités cognitives en lecture pour adultes (ECCLA), Rapport du contrat État-Région, 1993 (édité par le CNDP).

Permet la mise en évidence des difficultés de lecture afin de proposer une remédiation adaptée. Une étude de la DEP, direction de l'évaluation et de la prospective, en collaboration avec l'Inspection générale et les Directions pédagogiques du ministère détermine plusieurs niveaux de lecture (compétences de base, approfondies et remarquables). Voir notamment les notes d'information citées ci-après qui en rendent compte :

Note d'information 94-11: Les compétences en lecture et en calcul des élèves à l'entrée en CE2 et en sixième.

Note d'information 94-12: Évolution des performances en français et en mathématiques à l'entrée en sixième entre 1980 et 1993.

Note d'information 93-20: Savoir lire et aimer lire : une relation assez souple.

\section{Troubles de la lecture}

Comprendre le « pathologique » peut permettre de mieux saisir les caractéristiques du «normal ».

GELBERT Gisèle, Lire, c'est vivre: comprendre et traiter les troubles de la parole, de la lecture et de l'écriture, Paris, éd. Odile Jacob, 1994, 198 p.

Cet ouvrage propose des parcours de rééducation d'enfants non-lecteurs. L'auteur propose une représentation des fonctions linguistiques qui relient le niveau sensoriel acoustico-phonatoire-graphique, les représentations mentales et le sémantisme conceptuel. En partant de "préalables" (sorte de verrou dont la levée conditionne l'ouverture de tout le système linguistique), l'auteur montre comment lutter contre les aphasies sans lésion. 
prioritairement constitué par les enseignants et les parents concernés. La dernière partie aborde les méthodologies d'apprentissage de la lecture et les actions de rééducation mises en place par l'Éducation nationale.

\section{Nous vous rappelons}

Une application informatisée des principes de la lecture rapide appliquée aux domaines de la formation et de l'école.

\section{RÉSUMÉS}

La bibliographie signale des références récentes. Les documents cités sont disponibles au CIEP et comportent des bibliographies qui complètent celle-ci.

\section{AUTEUR}

\section{MARIE-GENEVIÈVE PICART}

Centre international d'études pédagogiques (CIEP) 